\title{
COMPLETE AURICULO-VENTRICULAR DISSOCIATION WITH HIGH VENTRICULAR RATE IN PAROXYSMAL TACHYCARDIA
}

\author{
BY \\ CLIFFORD G. PARSONS
}

Received July 19, 1943

The ventricular rate in auriculo-ventricular dissociation is commonly less than 60 , and in 1923 Hewlett was able to find only 14 recorded instances in which this rate was exceeded. Most of these patients were under the influence of digitalis and all but one of the remainder had organic heart disease; the exception was a woman whose dissociation developed after tonsillectomy (White, 1916). In 'Hewlett's own case the P-R interval was considerably prolonged, and during attacks of dissociation the ventricles usually, although not invariably, beat more rapidly than the auricles; most reported examples which he quotes had auricular rates exceeding the ventricular. His observations led him to conclude that the occurrence of dissociation is favoured, on the one hand by the presence of partial A-V block or by the administration of digitalis, and on the other by factors tending either to decrease the auricular or to increase the ventricular rate. White's patient, for example, having recovered from a spontaneous attack of dissociation, would still develop the abnormal rhythm if the carotid sinuses were stimulated, the auricles slowing until the A-V node escaped. Hewlett felt that the rapid ventricular rate in his own patient, together with the conspicuous effect on the rhythm of changing vagal tone, were evidence that the pacemaker lay in the A-V node.

Cowan (1939) reviewed ten cases of dissociation and of these only two had sound hearts, both having a slow ventricular rhythm; all ten had ventricles beating either at the same rate or rather faster than the auricles. This point is emphasized by Katz (1941) for distinguishing between dissociation, which he regards as a functional condition, and heart block, which is frequently due to organic disturbance. "In dissociation the rate of the ventricles is equal to or faster than that of the auricles, while in A-V block, with rare exceptions, the reverse is true."

Barker and his colleagues (1943), in an interesting paper, discuss the occurrence of A-V block in paroxysmal auricular tachycardia. After reviewing 17 previously reported cases they describe 18 of their own and compare the condition with auricular flutter, pointing out, amongst other features, that carotid sinus pressure slows the ventricles without influencing the auricular rate, and usually fails to arrest the attack. The degree of block encountered ranged from partial block with occasional dropped beats, through $2: 1$ and higher grades of $\mathrm{A}-\mathrm{V}$ block, to two examples of complete heart block. These last two patients had regular ventricular rates of 84 and 26 respectively; one, a woman aged 26 , had organic heart disease, and the other was a man of 70, reported by Singer and Winterberg (1922).

A somewhat different abnormality of rhythm was described by Barker (1924), who reported a case of paroxysmal auricular tachycardia in which an attack was disturbed by a brief phase of ventricular tachycardia. The ventricular contractions were slightly irregular and no auricular complexes could be identified. The patient had had attacks of palpitations for 36 years and her heart was otherwise sound.

The report which follows concerns a patient subject to attacks of paroxysmal auricular tachycardia. She had no evidence of organic heart disease and had had no drugs. A spontaneous, short-lived attack in which auricles and ventricles beat quite independently, each at a rate well in excess of 100 , was recorded during her first appearance at hospital. 


\section{Case Report and Electrocardiograms}

A woman of 29 attended hospital in November 1942, complaining of attacks of palpitation which she had had for 7 years. The paroxysms occurred two or three times a day, always started and ended abruptly, lasted from ten minutes to two hours, and were precipitated especially by emotional upsets. She was a well-built, healthy woman with an apparently normal heart. There were no signs of hyperthyroidism and the only focus of infection lay in some doubtful teeth which were removed later without influencing her attacks. Blood count, sedimentation rate, and X-ray

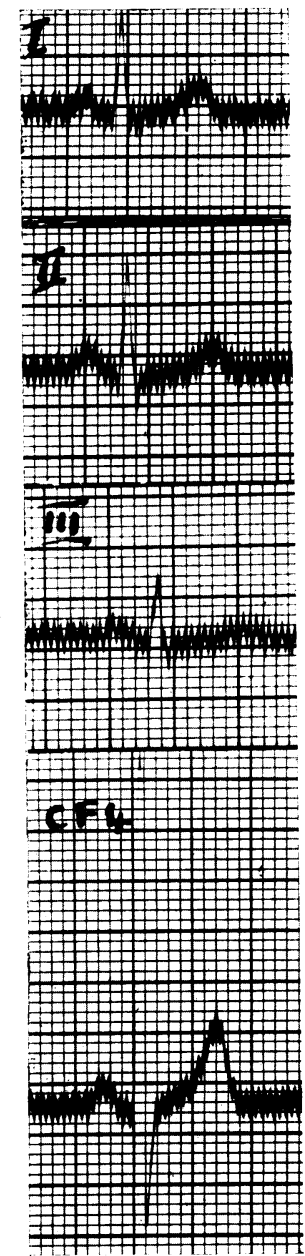

Fig. 1.-Sinus rhythm. of chest were all normal.

She was not unduly distressed during an attack of tachycardia; her pulse rate was 136, quite regular, and uninfluenced, clinically, by exercise or carotid sinus pressure. A triple venous pulse wave was visible at the root of the neck and the heart sounds were normal apart from their rapidity.

Electrocardiograms taken during sinus rhythm (Fig. 1) are normal in every respect. The $\mathbf{P}-\mathbf{R}$ interval has been measured on numerous occasions and varies between $0 \cdot 14$ and $0 \cdot 16 \mathrm{sec}$. Many records have been taken in the course of her paroxysms of tachycardia and practically no variation has been seen. The attack invariably starts with a premature $\mathbf{P}$ wave lying early in the descending limb of the preceding $T$; during the paroxysm $P$ and $T$ waves are superimposed and the sequence ends with a compensatory pause before the resumption of sinus rhythm (Fig. 2). The heart rate in these episodes is 136 and the P-R interval (which in this and subsequent records is measured from the summit of $P$ to the summit of $R$ ) is slightly longer than in sinus rhythm, i.e. $0 \cdot 16-0 \cdot 18 \mathrm{sec}$.

Occasional isolated extrasystoles, which resemble the first beat of the tachycardia, have been observed and from the appearance of these complexes it would seem that the excitable focus must lie either in, or very close to, the S-A node.

Fig. 3 shows the first cardiogram obtained from the patient. In the limb leads the auricles are beating regularly at a rate of 152 whilst the ventricules have assumed an independent rhythm which varies between 113 and 136. The $\mathbf{P}$ waves in leads I and III are rather larger than those in records of sinus rhythm, and this, combined with the fact that their rate is greater than that in paroxysms of auricular tachycardia, may mean that a kind of flutter has developed. The QRS complexes are of supraventricular type and do not differ materially from those seen in other tracings, but the $\mathrm{T}$ waves are inconspicuous. The form and rate of the ventricular waves suggest that they arise in or near the A-V node. In $\mathrm{CF}_{4}$ the rhythm is much the same as in the limb leads, but the auricular rate is a little slower and there may conceivably be conduction from auricles to ventricles, anyway after the first, seventh, and eleventh auricular contractions. $\mathrm{CF}_{1}$, taken a few seconds later, opens with what seems to be the last beat of a paroxysm of auricular tachycardia; this is followed by two sinus beats, a short paroxysm of auricular tachycardia and finally by normal sinus rhythm.

The patient would not come into hospital for investigation and the following observations were therefore carried out in the out-patient department. They were kindly made for me by Dr. D. R. Humphreys working in Professor K. D. Wilkinson's clinic at the Queen Elizabeth.Hospital, Birmingham.

Measurements reproduced in the various figures which follow are derived from naked eye examination without the aid of a comparator. They are therefore not strictly accurate but may serve to

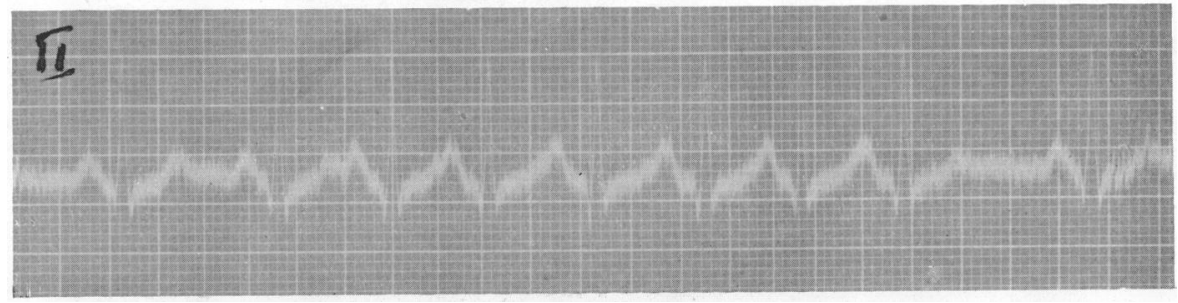

Fig. 2. - Onset and ending of a paroxysm of auricular tachycardia. 


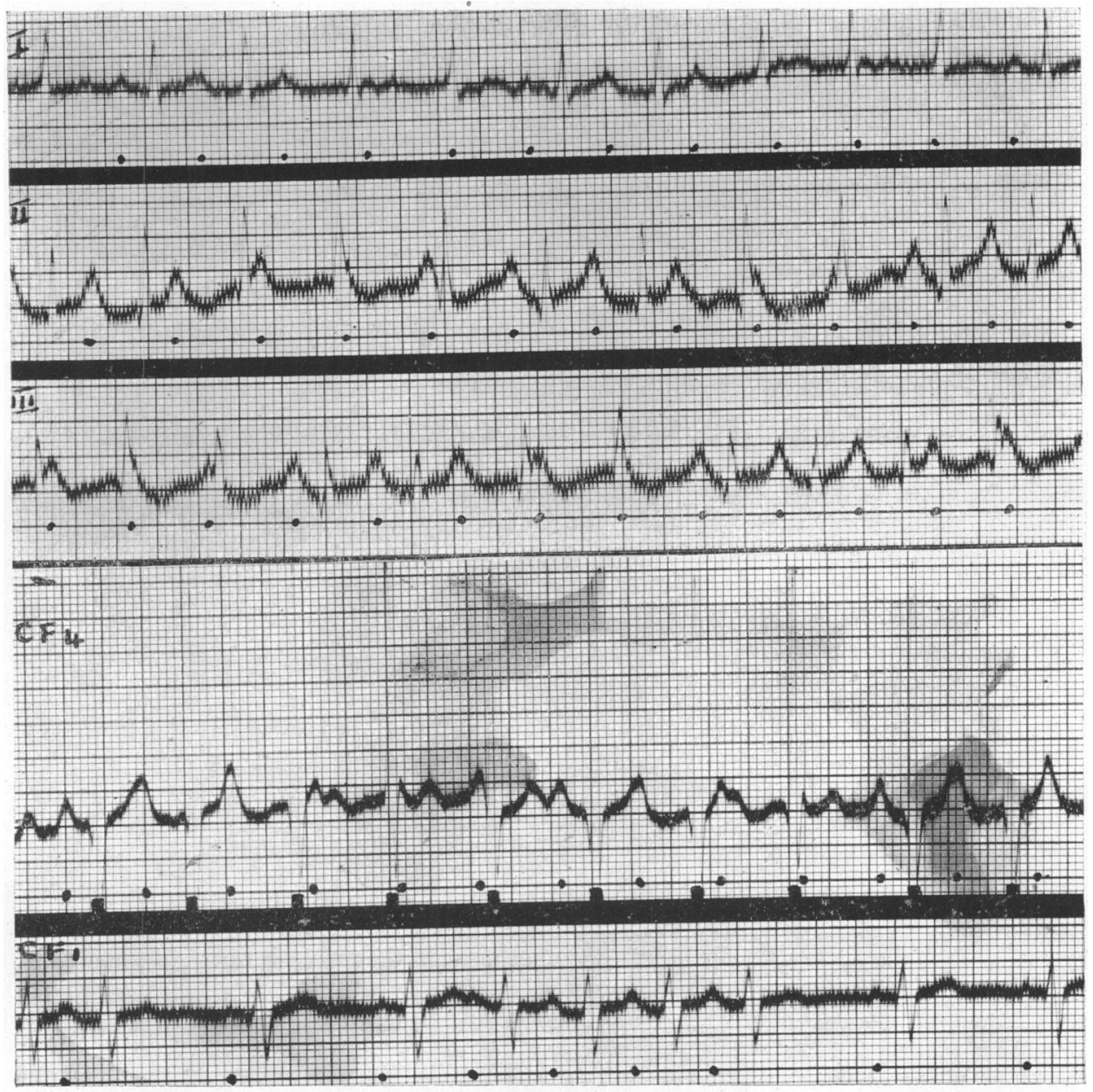

FIG. 3.-Cardiogram showing complete dissociation between auricles (marked with dots) and ventricles, in leads I, II, III, and probably in $\mathrm{CF}_{4}$. $\mathrm{CF}_{1}$ shows paroxysms of tachycardia and normal sinus beats.

emphasize the points brought out by the records. Exercise had absolutely no effect on the rate or form of a paroxysm of auricular tachycardia. Pressure on the left carotid sinus or on both sinuses simultaneously led to the resumption of sinus rhythm for a single beat (Fig. 4). The auricular rate continues practically unchanged whilst the ventricles beat more slowly. The last visible $P$ wave before the sinus contraction is smaller than its predecessors, but it falls in front of, instead of on, a $\mathrm{T}$ wave and it does not differ materially from the $\mathbf{P}$ of the sinus beat. The next $\mathbf{P}$ wave is lost; it is perhaps directly superimposed on the QRS as the voltage of that complex is a little greater than the average. The $T$ wave in the ventricular beat resembles that of the preceding complex, being much less conspicuous than its successor. The $\mathbf{S}-\mathrm{T}$ interval is slightly depressed.

Lewis (1925) has pointed out that stimulation of the left carotid sinus will often decrease the speed of conduction from auricle to ventricle. If it is assumed that the missing auricular wave in Fig. 4 is buried in the ventricular beat, this may be an example of the Wenckebach phenomenon. The length of successive $P-R$ intervals, measuring 0.16 sec. in the early beats, increases to $0.18,0.30$ and 0.39 before a beat is dropped and sinus rhythm is restored. The successive increments are therefore $0.02,0 \cdot 12$ and 0.09 , the first of which might perhaps be ignored. Further discussion of this subject follows later.

Five minutes after the sinus compression experiment, two minims of adrenalin $(1: 1000)$ were injected intravenously, in an attempt to stimulate the sympathetic. The patient became pale, shaky and apprehensive, with rather a feeble pulse. At the same time some interesting changes developed in the electrocardiograms. Fig. 5 is a section of the tracing taken immediately after injection. It shows two runs of tachycardia separated by a sinus contraction. The auricles continue to contract regularly at the paroxysmal rate of 136 , whilst the ventricular rate slows. The last visible $P$ wave in both of the abnormal sequences is small, but this can be explained by the fact that they are not directly superimposed on $\mathrm{T}$ waves (a small $\mathrm{T}$ is visible in the two beats before the sinus contraction). It is possible 


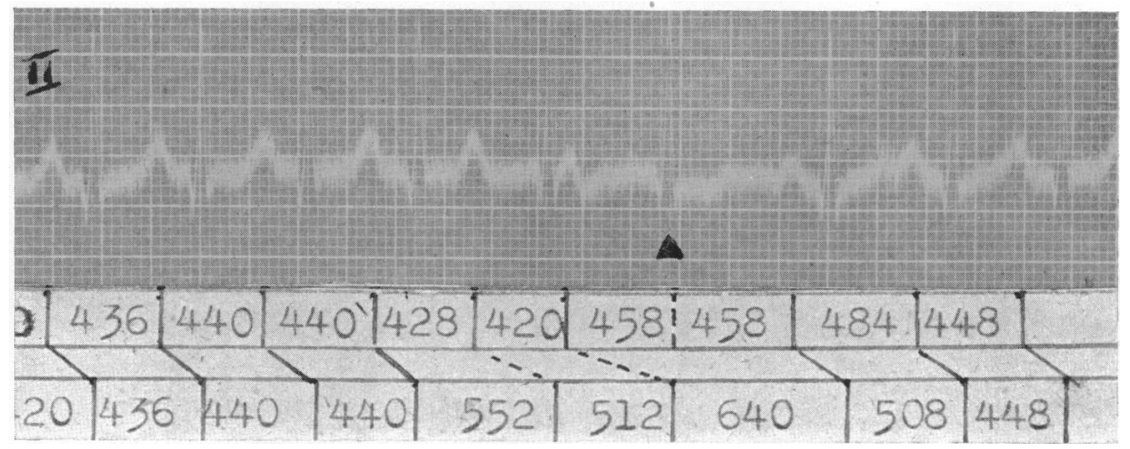

FIG. 4.-Effect of compression of the left carotid sinus during an attack of paroxysmal tachycardia.

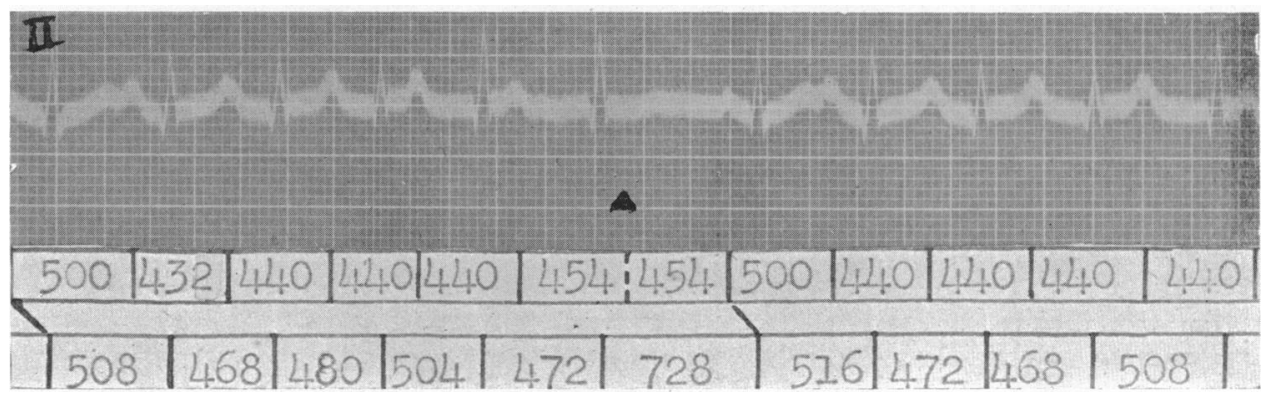

Fig. 5.-Paroxysm of tachycardia immediately after administration of adrenalin.

that a $\mathbf{P}$ wave falls on the final QRS of the first sequence, but if this is so the auricular contraction must be somewhat premature. The QRS waves in this tracing vary considerably in voltage but are obviously smaller than the average throughout. In other respects the record is not unlike an exaggerated version of that obtained by carotid sinus pressure.

Half a minute afterwards (Fig. 6), the same effect is still present, with the auricles beating regularly

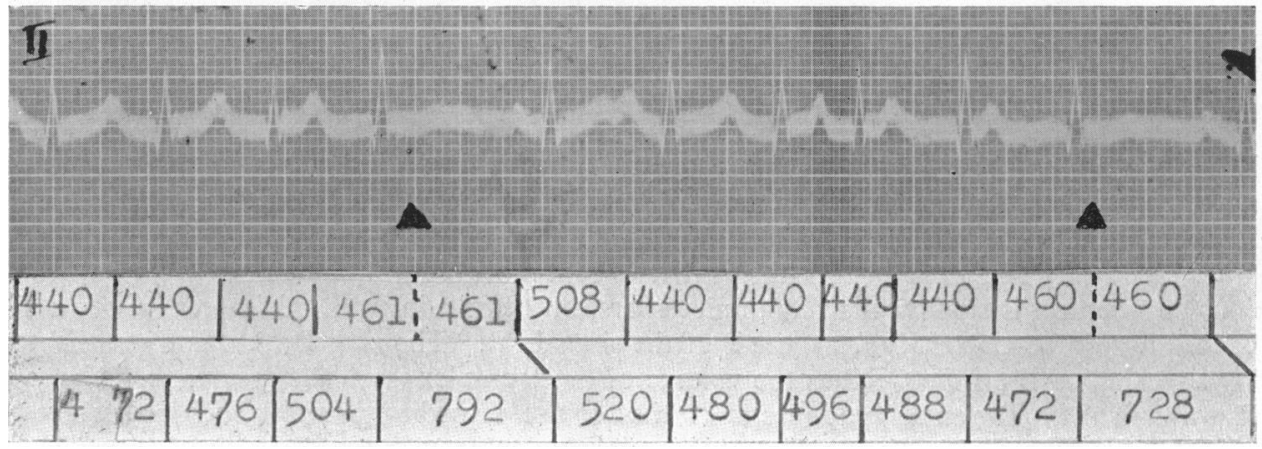

Fig. 6.-Approximately half a minute after adrenalin.

at a rate of 136 and the ventricles lagging behind. The last $P$ seen in the first sequence does not differ from those which go before it; presumably it is superimposed on a $T$ wave. In the second sequence the final $\mathbf{P}$ is of lower voltage, but it lies just before a small $\mathrm{T}$ wave. It should be noted that the third $P$ wave of this sequence differs in shape from the others because of a momentary sticking of the film in the camera. A recurrence of this mechanical defect mars several parts of the continuous record taken throughout this experiment and makes measurements impossible in some places.

The section of film shown in Fig. 7 was taken about a minute after the exhibition of adrenalin and again demonstrates essentially the same features. By the end of the second minute (Fig. 8) the effect of the drug is beginning to wear off, but the same tendency is still recognizable, the auricles continuing at their old rate of 136 and the ventricles beating rather more slowly. The shape and size of $P$ waves is to all intents and purposes constant and for a $\mathbf{P}$ wave to be superimposed on the QRS before the sinus beat, considerable prematurity would be necessary. 


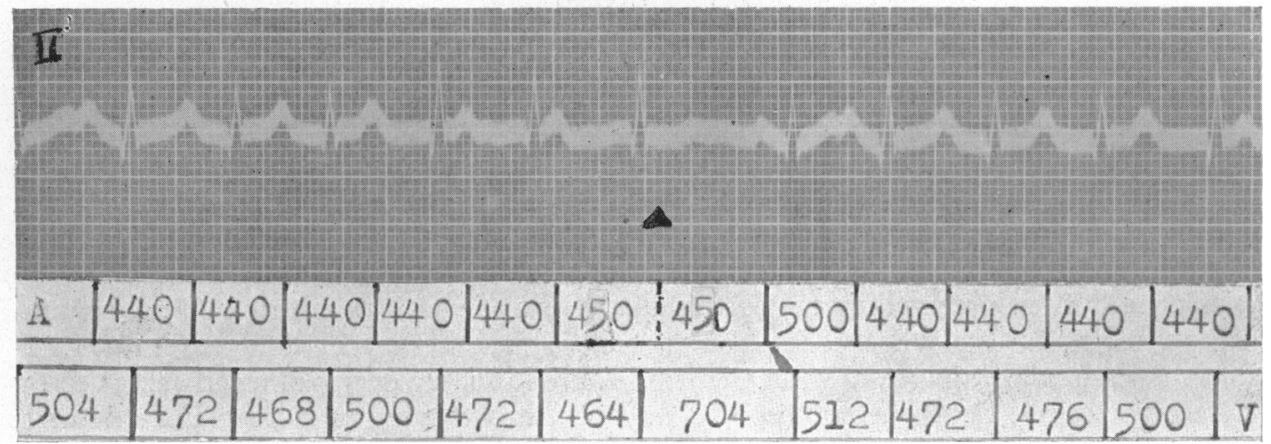

FIG. 7.-One minute after adrenalin.

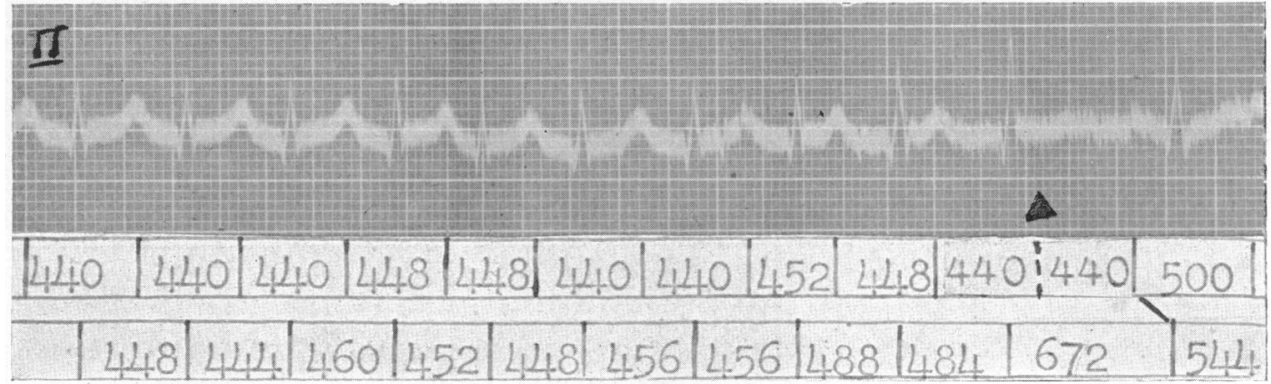

FIG. 8.-Two minutes after adrenalin.

Three quarters of an hour after giving the adrenalin an attempt was made to lower vagal tone by the injection of atropine (gr. 1/100) subcutaneously. Unfortunately the continuous film ran out at this point, but Fig. 9 (upper half) shows the state of affairs in lead I, 15 minutes later. The auricles

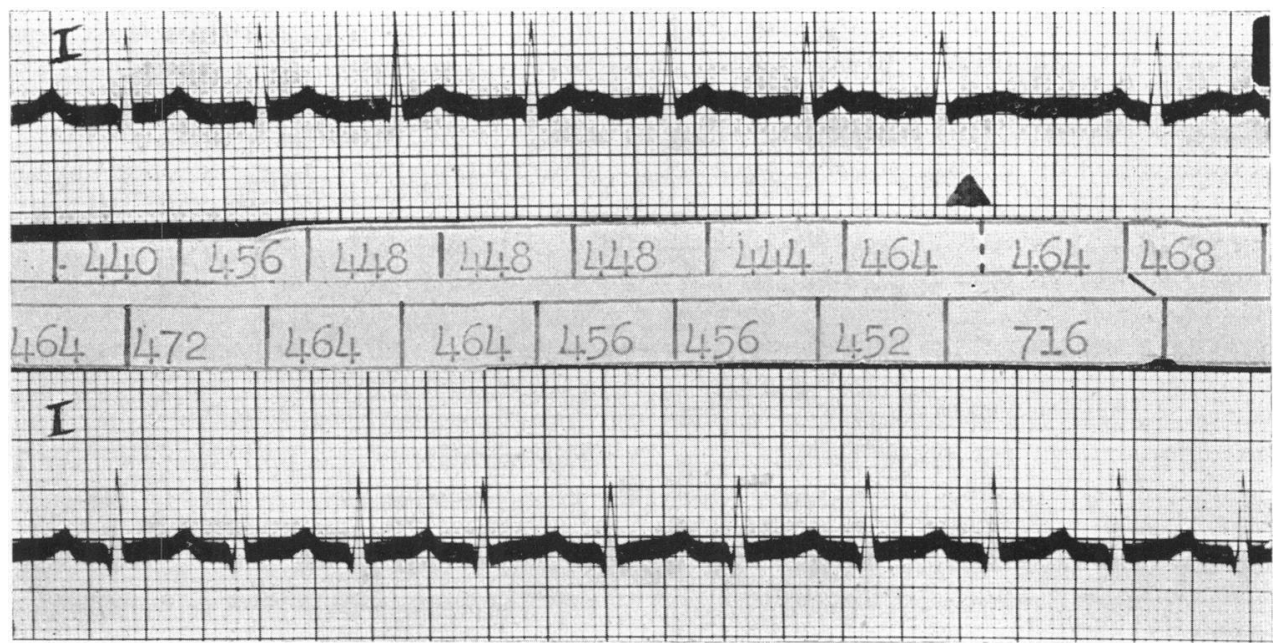

FIG. 9.-Twenty minutes after atropine.

are beating a little more slowly than in previous tracings but the ventricles are still slower than the auricles. The general features are the same as those already described after the injection of adrenalin, but in this film $\mathrm{P}$ and $\mathrm{T}$ waves are more readily distinguished. The QRS preceding the sinus contraction is not greater in voltage than the other waves of the curve and it seems unlikely that a $\mathbf{P}$ wave is incorporated in it. The sinus beat is followed by a premature $\mathbf{P}$, lying in the descending limb of $\mathrm{T}$, and almost certainly marks the initiation of the paroxysm of auricular tachycardia which is shown in the lower tracing, taken a few seconds later. The rate in this paroxysm is 141 . 


\section{DisCUSSION}

Adrenalin, atropine, and probably carotid sinus pressure have all produced the same effect on a paroxysm of tachycardia. Lewis (1925) states that adrenalin, besides stimulating the sympathetic nerve endings, also acts directly on the vagal nerve centres in the medulla. Cushny (1936) agrees that the vagal centres may be stimulated, although he argues that it is not a direct action but a reflex stimulation following a rise of blood pressure. He points out too, as other writers have done, that atropine given in small amounts may activate instead of depressing the tenth nerve. It is probable, therefore, that the similar effects of all three experiments is due in each case to stimulation of the vagus.

It has already been observed that vagal stimulation may diminish the speed of conduction from auricles to ventricles. The Wenckebach phenomenon is characterized by three main features. The P-R interval progressively increases in length with each beat but the degree of lengthening decreases in successive complexes. Consequently the $\mathbf{R}-\mathbf{R}$ intervals tend to become shorter and shorter until a beat is dropped. During the sequence auricular complexes are followed by ventricular complexes until the ventricle defaults, leaving an isolated $P$ wave before the next sinus beat. Campbell (1943) has demonstrated, however, that the rule of $\mathbf{P}-\mathbf{R}$ increments is not invariable and that occasionally the increase of the third interval over the second is greater than that of the second over the first.

Allowing both for Campbell's findings and for the fact that measurements taken from the tracings. are not strictly accurate, it remains difficult to reconcile the figures (summarized in the accompanying table) with a diagnosis of partial heart block. Moreover in some of the cardiograms the R-R intervals tend to increase instead of diminishing with each successive beat.

Table Showing P-R Intervals and Increments Measured in Thousandths of a Second

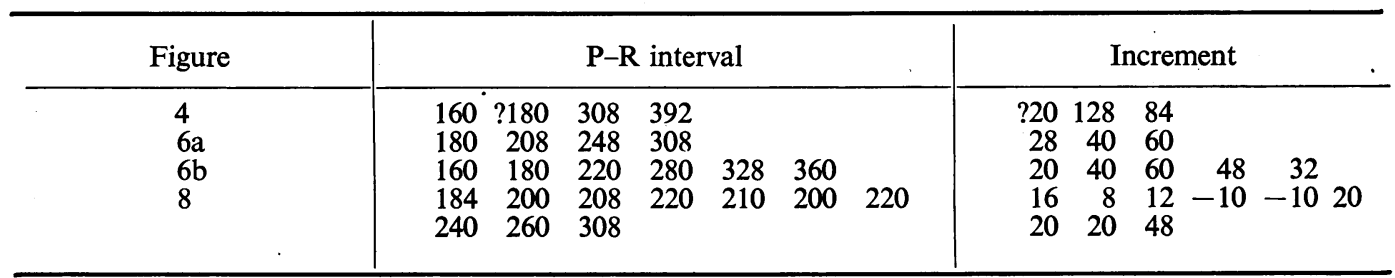

The argument that partial heart block is responsible for the abnormal rhythm shown in Fig. 4-9 is in any case based on an assumption that seems quite unwarranted. It has been pointed out that in partial block the last wave before the sinus beat should be auricular. In none of the tracings is this final $\mathbf{P}$ wave visible and it has to be assumed therefore that it is superimposed on a QRS complex. This necessitates the further assumption that the quite regular auricular rhythm is broken, for in practically every instance the $P$ wave would have to be slightly premature to fall directly on the QRS. Black triangles on the figures show the points at which the $\mathbf{P}$ waves would have fallen if the auricles had continued at their original rate. It is unbelievable that a premature $\mathbf{P}$ wave could fall with such exactness on a QRS on so many occasions. Consequently it is most improbable that this is an example of partial heart block with dropped beats, and if the condition is due to such a defect it is of most unusual type.

The changing shape of the $\mathbf{P}$ waves may suggest that there is a shift of the pacemaker towards the A-V node, but the alteration in size is also explicable by the relationship of the $P$ and $T$ waves, $P$ being smaller only when the two fail to coincide. This is made the more probable explanation because in some of the tracings (Fig. 6 and 8 ) there is no change in the size of the $\mathbf{P}$ wave. The progressive lengthening of the $\mathrm{P}-\mathrm{R}$ interval is a further argument against this diagnosis.

A third, and more probable, interpretation of the abnormal rhythm produced by vagal excitation is that there are short runs of complete A-V dissociation linked by isolated sinus beats. This would be in keeping with the spontaneous attack of dissociation illustrated in Fig. 3, especially if it is allowed that there are occasional sinus beats in $\mathrm{CF}_{4}$. The development of the independent auricular and ventricular rhythms must be based on factors quite different from those which caused dissociation in White's (1916) case, or those mentioned by Hewlett (1923), for in the present instance the auricular rate continues unchanged and it is the ventricles that beat more slowly. It is likely that the auricles are driven by an excitable focus lying in or near the S-A node, a focus which is usually responsible for paroxysms of auricular tachycardia. In certain circumstances a barrier develops in the region of the A-V node, preventing the passage of impulses to the bundle. When this barrier is present the ventricular rhythm is probably governed by a second pacemaker lying in the neighbourhood of the A-V node. This pacemaker, having been sensitized to a high speed of activity by a paroxysm of auricular tachycardia, continues to send out impulses at a rapid rate, and so there develops a simultaneous auricular and nodal tachycardia. 


\section{A-V DISSOCIATION IN PAROXYSMAL TACHYCARDIA}

Some light is thrown on the nature of the A-V nodal barrier by the effect of vagal stimulation during attacks of auricular tachycardia. The dissociation produced is then intermittent, with a sinus beat separating one period of block from the next. The sinus beat is always preceded by a dropped auricular beat. This suggests that the relatively long period of auricular inactivity allows sufficient recovery in the area around the A-V node for the next auricular impulse to follow its normal path to the ventricle. The passage of this solitary contraction wave again disarranges the mechanism and leaves a barrier which persists until the auricles once more default. The defect probably results from an abnormally long refractory period in the area concerned and is presumably related to the mechanism responsible for the Wenckebach phenomenon.

If the rhythm in all the records is in fact due to dissociation, it becomes difficult to decide at what point, following carotid sinus pressure (Fig. 4), conduction gives way to the two independent rhythms. It happens that this is the one electrocardiogram in which the Wenckebach phenomenon may be present, for it is easy to believe that the missing $P$ is superimposed on a QRS. If, however, dissociation is present it probably develops at the fifth beat, where there is a sudden lagging of the ventricle. It will be noted that, as observed by Barker and his colleagues (1943), carotid sinus pressure fails to arrest the attack, but slows the ventricles.

Cardiograms not unlike the record in Fig. 3 were obtained from three of the patients described in Barker's paper (Fig. 7A, 8B, and 11B). These are considered to be examples of partial heart block, but the mechanism of impaired conduction is not discussed. The irregular rhythm of the ventricles is presumably ascribed to prolonged conduction from auricles to ventricles, but the variations in the $\mathbf{P}-\mathbf{R}$ intervals are considerable and in the short illustrative tracings follow no obvious pattern, though such a pattern may perhaps be apparent in longer records. As far as can be seen the block is not of the Wenckebach type and is probably related to the mechanism described above. In this connection it is interesting that temporary nodal rhythm was observed in two of these cases. All three patients were receiving digitalis; two had organic heart disease and the third had an enlarged heart and, during attacks of tachycardia, a high blood pressure. The auricular rates in the illustrative records were 212,188 , and 167 with corresponding ventricular rates of 165,112 , and 131 .

One other patient relevant to the discussion (Case 9) was a woman suffering from chronic rheumatic heart disease who, when under the influence of digitalis, developed tachycardia with partial block. Exercise increased the auricular rate slightly and " . . . the ventricles responded to each auricular beat for a short time, then became slower and irregular and finally displayed a long period of near standstill, interrupted by idiopathic ventricular beats. . . ." These idiopathic complexes are similar in structure to the complexes throughout the record, suggesting a point of origin close to the A-V node. This makes it clear that, in some cases of paroxysmal tachycardia, the auricular impulses may fail partly or completely to stimulate the ventricles, and that there is a tendency for the ventricles to be controlled by a pacemaker lying in or near the A-V node, the impulses from which are not necessarily regular.

On account of wartime conditions a thorough search of the literature has been impossible, but a limited review has revealed no other case in which the particular form of dissociation here described has developed in a patient without organic heart disease and not under the influence of digitalis.

\section{SUMMARY}

A case of paroxysmal auricular tachycardia is described, in which there developed a spontaneous attack of A-V dissociation, with high auricular and ventricular rates amounting to a double tachycardia. The condition could be partly reproduced during attacks of auricular tachycardia by atropine, adrenalin, and carotid sinus pressure. The patient's heart was otherwise normal and no drugs had been given. It is suggested that the condition is due to a form of heart block the mechanism of which is allied to, but not identical with, the Wenckebach phenomenon.

\section{REFERENCES}

Barker, P. S. (1924). Heart, 11, 67.

Barker, P. S., Wilson, N. W., Johnston, F. D., and Wishart, S. W. (1943). Amer. Heart J., 25, 765.

Campbell, M. (1943). Brit. Heart J., 5, 55.

Cowan, J. (1939). Ibid., $1,3$.

Cushny, A. R. (1936). Textbook of Pharmocology and Therapeutics. Revised by Edmunds, C. W., and Gunn, J. A. Churchill, London.

Hewlett, A. W. (1923). Heart, 10, 9.

Katz, L. N. (1941). Electrocardiography. Kimpton, London.

Lewis, T. (1925). Mechanism of the Heart Beat. London.

Singer, R., and Winterberg, H. (1922). Wien. Arch. inn. Med., 3, 329.

White, P. D. (1916). Arch. intern. Med., 18, 243. 